\title{
Mixed Weibull Distribution Model of DC Dielectric Breakdowns with Dual Defect Modes
}

\author{
Allen Andersen and JR Dennison \\ Materials Physics Group \\ Utah State University \\ 4415 Old Main Hill, Logan, UT 84322 USA \\ allen.andersen@aggiemail.usu.edu,jr.dennison@usu.edu
}

\begin{abstract}
This work provides physical insight into common statistical models for DC dielectric breakdown field strengths. Voltage step-up tests were performed on low density polyethylene films. The merits of generalizations to widely-used empirical Weibull models are discussed. The cumulative probability distributions of the breakdown fields were fit to standard two- and three-parameter Weibull distributions. Mixed two-parameter Weibull distributions, sometimes used in the literature to model multiple breakdown modes, were found to yield the best fits to the data. In addition, the same data were fit to a physically-motivated dual-defect mean field model incorporating both low- and highenergy defect modes with different defect densities; this produced a much better fit than single-defect mean field models. Values obtained for the mean defect energies and densities were within the ranges expected from independent determinations of these intrinsic materials properties. By incorporating these physicsbased concepts into traditionally empirical models, their accuracy and utility can be extended. The mixed Weibull distribution and the dual-defect model predicted very similar cumulative distributions of LDPE breakdown data, suggesting that mixed Weibull distributions may reflect similar multiple defect modes used in dual-defect models. Theories of DC breakdown, based on distributions of microscopic defects in disordered insulating materials may provide improved guidance in understanding the physical origins of empirical parameters used in statistical methods to characterize breakdown properties.
\end{abstract}

Keywords-breakdown; electrostatic discharge; polymers; Weibull

\section{INTRODUCTION}

The stochastic nature of electrostatic breakdown in highly disordered insulating materials (HDIM) continues to present a challenge in both theory and application [1]. Traditionally, the statistical Weibull distribution and its variants have been used to describe both the centroid and the width of the measured distribution of breakdown electrostatic field strength [2]. Although such distributions provide reasonable empirical fits, there is debate as to how-or even if-fitting parameters of the Weibull distributions correspond to physical properties of a test material [3]. While simple approximations have led to basic physical models of electrostatic discharge (ESD) [4-6], empirical models are necessitated by the difficulty in reliable physical descriptions of breakdown processes in HDIM. This paper describes how insights from simple physical models can be extended to explain some characteristics of the empirical fits.

Research was funded by a NASA Space Technology Research Fellowship

\section{WEIBULL STATISTICS}

Weibull functions are often used to describe the probability of dielectric failure due to increasing stress factors. In this study, it models the increasing probability of failure due to ESD with increasing applied field. As with other probability distributions, such as Gaussians, Weibull distributions are characterized by a centroid and a width parameter. In this section, the empirical cumulative distribution (ECD) of breakdown data is fit to two-, three-, and five-parameter Weibull functions, using methods similar to prior studies $[2,7,8]$. The data set fit illustrated here is comprised of 88 voltage step-up to breakdown tests on lowdensity polyethylene (LDPE); as described in greater detail in $[6,9]$.

\section{A. Two-Parameter Weibull}

The simplest Weibull function of field $F$ has only two parameters, $F_{0}$, the field corresponding to a $63.2 \%$ probability of breakdown, and a width parameter, $\beta$ :

$$
P(F)=1-\exp \left(-\left[\frac{F}{F_{0}}\right]^{\beta}\right) \text {. }
$$

It is straightforward to linearize this function with the transformation [7]

$$
x=\log (F) \text { and } y=\log \left[\ln \left(\frac{1}{1-P}\right)\right] .
$$

One then transforms the ECD of a data set in the same way, and, by fitting it to a line, the Weibull parameters can be extracted. The transformed data and corresponding fit are shown in Fig. 1. Fig. 1 (a) is a linear fit to all the data, while Fig. 2 (b) is a linear fit to the data, excluding data below a field of $200 \mathrm{MV} / \mathrm{m}$. Upon linearization, it appears that the first seven low-probability events at low fields do not follow the same trend as the rest of the data. The bands around the data indicate the ECD of the transformation evaluated at plus and minus the average of the standard deviations of the data. This is clearly an overestimation of the uncertainty; however, it demonstrates that the ECD is well defined.

It is apparent that only considering points above the minimum field threshold results in a better fit to the twoparameter Weibull model. This could be the result of extrinsic imperfections in some samples or tests (e.g., sample damage or impurities or contamination) resulting in a breakdown at an 

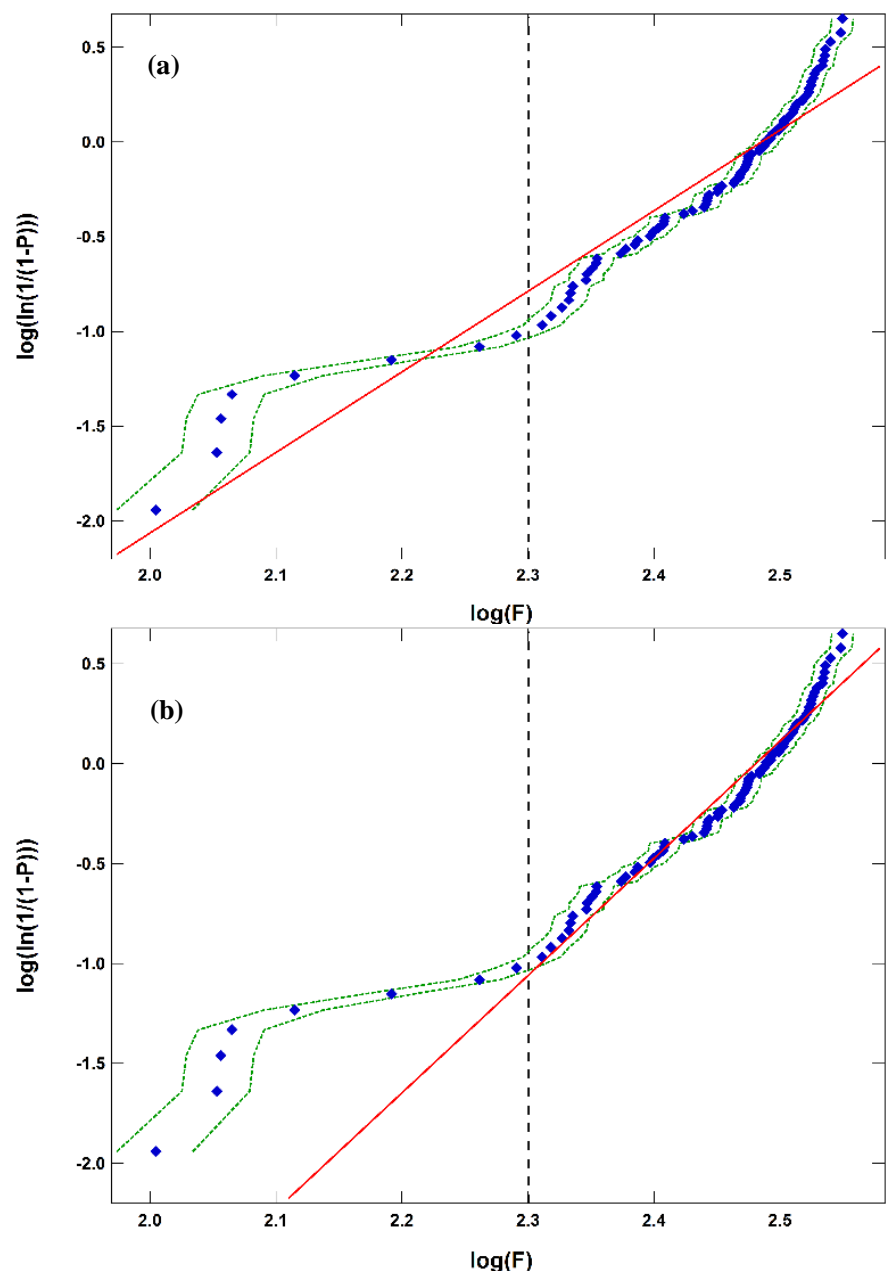

Fig. 1. The empirical cumulative distribution of breakdown data in LDPE transformed using (2). (a) Linear fit to all data. (b) Linear only to data above a minimum field, $200 \mathrm{MV} / \mathrm{m}$, as indicated by the vertical dashed line. Bands (dashed green lines) around the data indicate \pm the average of the standard deviations.

unusually low field. Alternatively, it might also be an indication in shortcomings in the model, as discussed below.

\section{B. Three-Parameter Weibull}

Equation (1) assumes that the probability of breakdown reaches zero as the field goes to zero. There are indications that there is a non-zero threshold field for breakdowns [3, 6, 7, 9, 10]. Such a threshold is incorporated in dynamic breakdown models $[6,11,12]$. Incorporating a threshold field into a Weibull distribution (1) requires a third parameter, $F_{s}$, and yields [7]

$$
P(F)=1-\exp \left(-\left[\frac{F-F_{S}}{F_{0}-F_{S}}\right]^{\beta}\right) \text {. }
$$

One can construct a transformation of (3) into a linear form similar to (2), namely [7]

$$
x=\log \left(F-F_{S}\right) \text { and } y=\log \left[\ln \left(\frac{1}{1-P}\right)\right] .
$$

Eq. (4) is not a unique transformation due to the reduction from three parameters to two in a linear transformation. One can iteratively optimize such a fit or, as done here, fit the untransformed data to (3) prior to transforming it. For our data set, the best fit was achieved with $F_{s}=0 \pm 90 \mathrm{MV} / \mathrm{m}$. This effectively reduces the three-parameter Weibull distribution (3) to the two-parameter Weibull distribution (1).

\section{Mixed Weibull Funtions}

Another common fitting method is to mix two or more Weibull distributions as a way to model multiple breakdown modes [2, 8]. Though this will lead to better fits, it is at the expense of an expanded parameter set. A recent study of a bimodal breakdown distribution in a LDPE nanodielectric composite material was fit to a two-parameter Weibull distribution [13].

In general, any number, $S$, of probability distributions can be mixed where the total probability function is

$$
P_{\text {mix }}(F)=\sum_{i=1}^{S} \frac{N_{i}}{N} P_{i}(F)
$$

where normalization requires

$$
\sum_{i=1}^{S} \frac{N_{i}}{N}=1
$$

For the case of $S=2$, (5) simplifies to

$$
P_{\text {mix }}(F)=p P_{1}(F)+(1-p) P_{2}(F),
$$

where $0 \leq p \leq 1$ satisfies (6). The mixture of two 2-parameter Weibull functions is therefore

$$
\begin{aligned}
P_{\text {mix }}(F)=p(1- & \left.\exp \left(-\left[\frac{F}{F_{01}}\right]^{\beta_{1}}\right)\right) \\
& +(1-p)\left(1-\exp \left(-\left[\frac{F}{F_{02}}\right]^{\beta_{2}}\right)\right)
\end{aligned}
$$

Here, $p$ represents the fractional weight of the first twoparameter Weibull distribution, $F_{01}$ and $F_{02}$ are their distribution centroids, and $\beta_{1}$ and $\beta_{2}$ are the corresponding width parameters.

There is no clear way to linearize (8), which is to be expected. If this is to be a better fit to the data, we would expect to see a mixture of two lines in the coordinate system used in Fig. 1. Again, the most straightforward method is to fit the data to the function in question, then transform it via (2) in order to

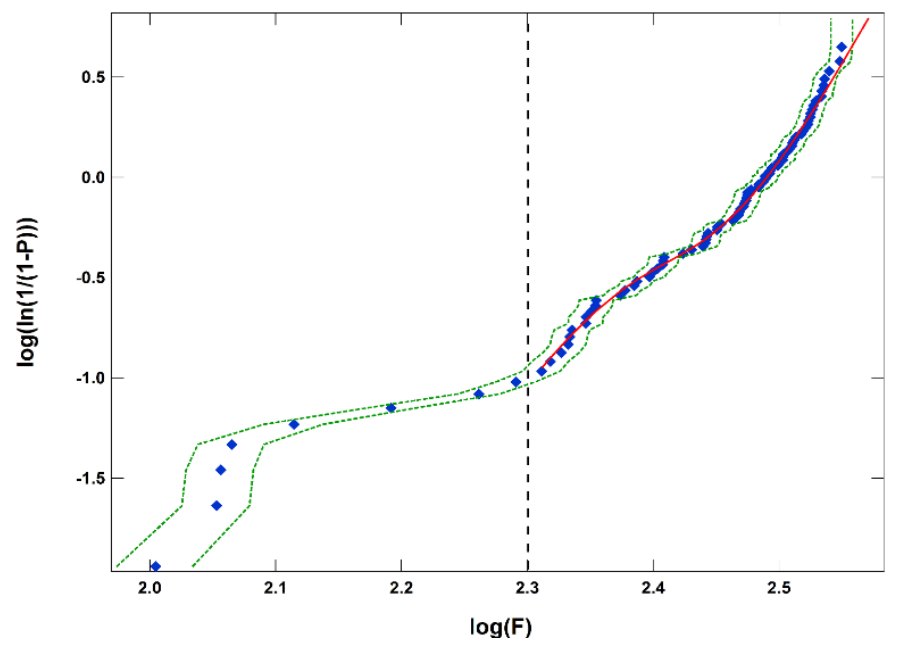

Fig. 2. The transformed mixed Weibull fit to the empirical cumulative distribution of breakdown data in LDPE for all points above the minimum field. 

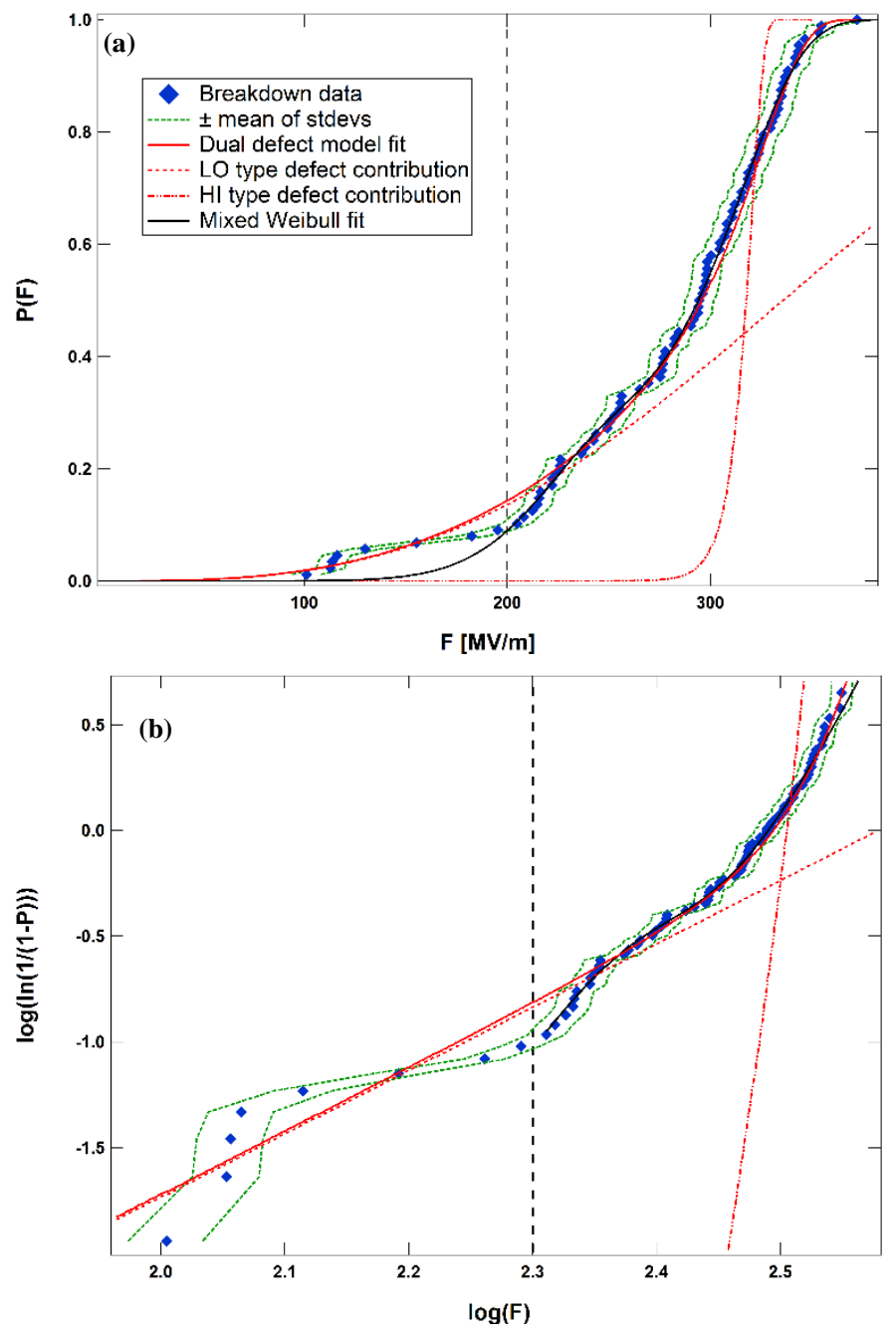

Fig. 3. A dual-defect model fit using (12-13) to the empirical cumulative distribution of breakdown data of LDPE. The contributions of the low-energy LO type and the high-energy HI type defects are shown individually by the dashed red curves. The black and green curves show the mixed Weibull fit and estimated uncertainties from Fig. 2 for comparison. (a) Untransformed axis. (b) Axis transformed by Eq. (2).

compare it to Fig. 1. This is shown in Fig. 2. Fitting, using the first 7 points or neglecting them, does not have a large impact since in each case the fits both miss these points and follow closely the remaining 81 points. Again, this could be either a shortfall of the model or indicative of sample impurities. In any case, it is clear that (8) is a better fit than (1).

\section{Defect DRIVEn Model}

Although, as shown here, mixed Weibull functions can provide a better fit to some data, it is difficult to infer any physical significance. It is plausible that the better fit is the result of simply adding more fitting parameter or perhaps there are indeed two breakdown modes or mechanisms requiring a mixture of two distributions. In either case there is not a straightforward way to extract intrinsic parameters of physical interest from the empirical fit [3]. We therefore turn to a simple, approximate model that incorporates physical parameters from the beginning.

\section{A. Single-Defect Model}

The simplest model of charge motion between defect states is the mean field theory, or frequently used Crine model [5]. This model assumes a single defect energy, $\Delta G_{d e f}$, with the defects spaced periodically with density $N_{\text {def }}$. Although this is obviously the incorrect picture for HDIM, it is the starting point for creating an approximate physical model. One can write the probability of one defect per activation volume as a function of field $F$ at temperature $T$ over elapsed time $t$ as [6]

$P_{d e f}(F, T, \Delta t)=\left(\frac{2 k_{B} T}{h / \Delta t}\right) \exp \left[\frac{-\Delta G_{d e f}}{k_{B} T}\right] \sinh \left[\frac{\varepsilon_{0} \varepsilon_{r} F^{2}}{2 k_{B} T N_{d e f}}\right]$.

The probability of a material surviving our step-up test is [6]

$$
\begin{aligned}
& P_{\text {survive }}^{\text {def }}\left(\frac{j \Delta V_{\text {step }}}{D}, N_{\text {step }}, T, \Delta t_{\text {step }}\right)= \\
& \quad \prod_{j=1}^{N_{\text {step }}}\left[1-P_{\text {def }}\left(\frac{j \Delta V_{\text {step }}}{D}, T, \Delta t_{\text {step }}\right)\right] .
\end{aligned}
$$

The probability of breakdown for a step-up test is then

$$
P_{\text {step }}^{\text {def }}=1-P_{\text {survive }}^{\text {def }} \text {. }
$$

The defect energy and density become the physically-motivated fitting parameters.

We were unable to find an acceptable fit to the entire LDPE data set with a single-defect model. The complicated nature of (11) precludes the use of generic fitting routines. However, an iterative manual approach quickly confirmed that, although (11) has the same general shape as the data and a Weibull distribution, the data do not fit well with physically reasonable values of the defect parameters. Separate fits to only low- and high-field data are somewhat more successful, as shown by the dashed red lines in of Fig. 3.

\section{B. Dual-Defect Model}

To obtain a better fit, (9) has been extended to model two defect species, termed LO and $\mathrm{HI}$, each with corresponding mean energies and densities [6]. The four-parameter dual-defect model is a simple sum of probabilities for each defect mode modeled by (9), namely

$$
\begin{aligned}
& P_{d e f}^{T o t}(F, T, \Delta t) \\
& =\left(\frac{2 k_{B} T}{h / \Delta t}\right) \sum_{i=L O, H I} \exp \left[\frac{-\Delta G_{d e f}^{i}}{k_{B} T}\right] \sinh \left[\frac{\varepsilon_{0} \varepsilon_{r} F^{2}}{2 k_{B} T N_{d e f}^{i}}\right] .
\end{aligned}
$$

Following the same logic in (10) and (11) with (12) substituted for (9) yields the dual-defect model of the step-up test [6].

$$
\begin{aligned}
& P_{\text {step }}^{\text {Tot }}\left(\frac{j \Delta V_{\text {step }}}{D}, N_{\text {step }}, T, \Delta t_{\text {step }}\right)= \\
& \quad 1-\prod_{j=1}^{N_{\text {step }}}\left[1-P_{\text {def }}^{T o t}\left(\frac{j \Delta V_{\text {step }}}{D}, T, \Delta t_{\text {step }}\right)\right] .
\end{aligned}
$$

LO type defects may correspond to lower-energy physical defects, such as polymer chain kinks or Kuhn pairs [6, 14, 15]. If $\Delta G_{L O}$ is not much larger than $k_{B} T$, then there is significant probability that these defects can be thermally annealed and can, therefore, be self-healing (although this is not yet incorporated into the model) [6]. HI type defects might correspond to higherenergy chemical defects, such as bond breaking [6]. Then $\Delta G_{H I} \gg k_{B} T$, and there would be no appreciable probability of 
recovery [6]. Realistically, we expect the defect energies must fall somewhere between $k_{B} T$ as a lower bound $(\sim 0.03 \mathrm{eV})$ and the dissociation energy of the strong C-C bonds in a polymer $(\sim 3.65 \mathrm{eV})$ as an upper bound $[6,16]$. Estimations of bond densities span orders of magnitude, but reasonable estimations of broken bond densities in LDPE are $\sim 10^{18}$ bonds $/ \mathrm{cm}^{3}$ and total available bonds are $\gtrsim 10^{22}$ bonds $/ \mathrm{cm}^{3}[6]$.

The fit in Fig. 3 was again obtained with an iterative fitting method due to the complexity of (13). The insets the individual contribution of both LO and HI defects are shown as well together with the mixed Weibull fit for comparison. As expected for fairly widely separated energies, the breakdowns probabilities at low fields are dominated by the low energy defects, effectively allowing for independent determination of the two energies.

The best fit LO defect energy $\left(\Delta G_{d e f}^{A}=0.89 \mathrm{eV}\right)$ and defect density $\left(N_{d e f}^{A}=2.4 \cdot 10^{21} \mathrm{~cm}^{-3}\right)$ are in very good agreement with bond-bending type defects [6]. The best fit HI defect density $\left(N_{d e f}^{B}=1.05 \cdot 10^{19} \mathrm{~cm}^{-3}\right)$ is close to that predicted for broken bonds. However, the best fit $\mathrm{HI}\left(\Delta G_{d e f}^{B}=1.1 \mathrm{eV}\right)$ defect energy is about a factor of 3 less than the C-C bond breaking. Nevertheless, the dual-defect model provides a much better fit with parameters that are within the range of reasonable physical values.

\section{CONCLUSION}

Dielectric breakdown in HDIM is an important yet difficult problem to model. For breakdowns in LDPE we have shown that a mixture of two Weibull functions is a better fit than a single Weibull function. We have also shown that a mean field theory incorporating two defect types is a better fit than a single-defect model. Comparison of the mixed Weibull empirical fit and the dual-defect Crine mean field model fit in Fig. 3 clearly shows they have the same basic shape and exhibit remarkable agreement over a wide range of fields. (Quantification of their agreement can be made through use of quantile-quantile plots [9]; indeed, minimization of the associated probability plot correlation coefficient may provide a method to calculate the best parameter set for one distribution given the parameter set for the other distribution.)

The Weibull function is much simpler than the physical model, but lacks a clear physical interpretation. The dual-defect model has physically motivated parameters, but at the cost of simplicity, even with the most basic assumptions. We propose that the dual-defect concept is reflected in the improved mixed Weibull fit, offering some physical insight to this empirical model.

In order to better establish the correlation between physical defects and the corresponding best Weibull fit, breakdown data for additional materials (polyimide, polypropylene, and borosilicate glass) are currently being acquired and will be modeled with both a mixed Weibull distribution and the dualdefect model. The results for glass may be particularly insightful, because higher energy bond-bending defects similar to those found in polymers are not expected. The dual-defect model should be extended to incorporate elements of dynamic breakdown models [11, 12], including threshold field values below which breakdown never occurs. Such thresholds might result from an equilibrium between low-energy defect creation and thermal annealing of these defects [5]. This may also facilitate clearer interpretation of the three-parameter Weibull parameter $F_{s}$ in terms of the threshold field [9].

\section{ACKNOWLEDGMENT}

We gratefully acknowledge insightful discussions with John Stevens and Dave Brown, as well as help with instrumentation and experiments from current and previous members of the Utah State University Materials Physics Group including Charles Sim, Dan Arnfield, and Anthony Thomas.

\section{REFERENCES}

[1] G. Teyssedre and C. Laurent, "Advances in high-field insulating polymeric materials over the past 50 years," Electrical Insulation Mag., vol 29, pp. 26-36, 2013.

[2] L. A. Dissado and J. C. Fothergill, Electrical Degradation and Breakdown in Polymers. London, UK: The Institution of Engineering and Tech., 1992.

[3] C. Laurent, C. Chauvet, and J. Berdala, "The significance of the Weibull threshold in short-term breakdown statistics," Dielectrics and Electrical Insulation, IEEE Trans., vol. 1, pp. 160-162, 1994.

[4] A. Miller and E. Abrahams, "Impurity Conduction at Low Concentrations," Phys. Rev., vol. 120, pp. 745-755, 1960.

[5] J.-P. Crine, J.-L. Parpal, and C. Dang, "A new approach to the electric aging of dielectrics," in Electrical Insulation and Dielectric Phenomena, 1989. Annual Report., Conf. on, 1989, pp. 161-167.

[6] A. Andersen, J. R. Dennison, A. M. Sim, and C. Sim, "Electrostatic Discharge and Endurance Time Measurements of Spacecraft Materials: A Defect-Driven Dynamic Model," IEEE Trans. Plasma Sci., in press.

[7] C. Chauvet and C. Laurent, "Weibull statistics in short-term dielectric breakdown of thin polyethylene films," Electrical Insulation, IEEE Trans. on, vol. 28, pp. 18-29, 1993.

[8] I. Rytöluoto, K. Lahti, M. Karttunen, and M. Koponen, "Large-area dielectric breakdown performance of polymer films - part i: measurement method evaluation and statistical considerations on area-dependence," Dielectrics and Electrical Insulation, IEEE Trans. on, vol. 22, pp. 689700, 2015.

[9] J. R. Dennison and A. Andersen, "Pre-Breakdown Arcing as Proxy for DC Dielectric Breakdown," in Electrical Insulation and Dielectric Phenomena (CEIDP), 2015 IEEE Conference on Ann Arbor, MI, 2015.

[10] A. Andersen and J. Dennison, "Pre-breakdown Arcing and Electrostatic Discharge in Dielectrics under High DC Electric Field Stress," 2014 Annual Report Conference on Electrical Insulation and Dielectric Phenomena, pp. 63-66, 2014

[11] T. Lewis, J. Llewellyn, M. Van der Sluijs, J. Freestone, and R. Hampton, "A new model for electrical ageing and breakdown in dielectrics," in Dielectric Materials, Measurements and Applications, Seventh International Conf. on (Conf. Publ. No. 430), 1996, pp. 220-224.

[12] C. Griffiths, J. Freestone, and R. Hampton, "Thermoelectric aging of cable grade XLPE," in Electrical Insulation, 1998. Conf. Record of the 1998 IEEE International Symp. on, 1998, pp. 578-582.

[13] Y. Wang, G. Chen, and A. Vaughan, "Space charge dynamics in silicabased polyethylene nanocomposites," in Electrical Insulation and Dielectric Phenomena (CEIDP), 2014 IEEE Conf. on, 2014, pp. 727-730.

[14] J. C. Anderson, K. D. Leaver, R. D. Rawlings, and P. S. Leevers, Materials science for engineers: CRC Press, 2004.

[15] K. C. Kao, Dielectric phenomena in solids: Academic press, 2004.

[16] P. Phillips, "Morphology and molecular structure of polymers and their dielectric behavior," Engineering Dielectrics Volume IIA Electrical Properties of Solid Insulating Materials: Molecular Structure and Electrical Behavior, p. 119, 1983. 